\title{
Association of CDKAL1 Polymorphisms with Early-Onset Atopic Dermatitis in Koreans
}

\author{
Won Il Heo, Kui Young Park, Mi-Kyung Lee ${ }^{1}$, Ju Hee Kim, Nam Ju Moon², Seong Jun Seo \\ Departments of Dermatology, ${ }^{1}$ Laboratory Medicine, and ${ }^{2}$ Ophthalmology, Chung-Ang University Hospital, Seoul, Korea
}

Background: Atopic dermatitis (AD) has increased in frequency to rates as high as $20 \%$ for children in developed countries. AD is one of the most common childhood diseases and has a complex etiology involving genetic and environmental factors. Thus, a broad understanding of genetic background is needed for early diagnosis of AD. Objective: Identification of candidate functional genetic variants associated with early-onset AD in Koreans. Methods: Wholeexome sequencing (WES) was performed in three families. Sanger sequencing was used to validate detected variants in 112 AD patients and 61 controls. Results: Functional variants were filtered by WES, and then variants related to allergic immune diseases were selected through a literature search. Two candidate non-synonymous single-nucleotide polymorphisms of CDKAL 1 (rs77152992) and ERBB2(rs1058808) were identified, c.1226C $>$ T, p.Pro409Leu, c.3463C $>$ G, and p. Pro1170Ala respectively. A case-control study was performed to determine whether rs77152992 and rs1058808 are candidate risk factors for early-onset AD. rs77152992 was significantly associated with early-onset AD (odds ratio [OR], 0.42; 95\% confidence interval $[\mathrm{Cl}], 0.21 \sim 0.83$; $p=0.0133$ ) in allele frequencies. The CC genotype of $C D K A L 1$ had significantly increased risk of AD (OR, 2.16; $95 \% \mathrm{Cl}, 1.0 \sim 4.6 ; p=0.0475)$. rs 1058808 had no correla-

Received July 20, 2017, Revised September 28, 2017, Accepted for publication October 25, 2017

Corresponding author: Seong Jun Seo, Department of Dermatology, Chung-Ang University Hospital, 102 Heukseok-ro, Dongjak-gu, Seoul 06973, Korea. Tel: 82-2-6299-1538, Fax: 82-2-6264-0129, E-mail: drseo@ cau.ac.kr

ORCID: https://orcid.org/0000-0003-2915-839X

This is an Open Access article distributed under the terms of the Creative Commons Attribution Non-Commercial License (http://creativecommons. org/licenses/by-nc/4.0) which permits unrestricted non-commercial use, distribution, and reproduction in any medium, provided the original work is properly cited.

Copyright $₫$ The Korean Dermatological Association and The Korean Society for Investigative Dermatology tion with $A D$. Total eosinophil count was significantly increased in AD patients with the CC genotype of CDKAL1 (rs77152992). Conclusion: CDKAL 1 (rs77152992) and ERBB2 (rs1058808) were deemed functionally interesting based on WES. Our case-control study suggests that the CC genotype of rs77152992 may be associated with increased eosinophil counts. It may enhance the risk of early-onset AD. (Ann Dermatol 30(3) 276 283, 2018)

\section{-Keywords-}

Atopic dermatitis, $C D K A L 1, E R B B 2$, Whole exome sequencing

\section{INTRODUCTION}

Atopic dermatitis (AD) is a chronic pruritic skin disease characterized by Th2-dominant inflammation including elevated serum immunoglobulin E (IgE) levels and peripheral eosinophilia, as well as abnormal barrier function. AD is one of the most common diseases in childhood and has a complex etiology involving genetic and environmental factors.

Family history has been reported as a risk factor for AD in large population studies ${ }^{1}$. Many other common and complex disorders have strong genetic heritable components as well ${ }^{2}$.

AD affects as many as $20 \%$ of children in developed countries $^{3}$, and various mutations of the filaggrin gene have been detected in about $30 \%$ of East Asian AD patients ${ }^{4}$. Filaggrin mutations can predict the severity of $\mathrm{AD}^{5}$. Thus, a broad understanding of the genetic background of this disease is needed for early diagnosis of AD, and for discovering specific therapies that will prevent the development of AD. Identification of genetic variants associated with common diseases and complex traits is required for 
early detection of AD.

Whole-exome sequencing (WES) has the attractive cost of sequencing better than next-generation sequencing, and can identify functional variants targeted to the exome, which is comprised of about 180,000 exons. Over $85 \%$ of pathogenic mutations occur in the protein-coding loci of genes ${ }^{6}$. WES has already been used to search and annotate variants through multiple analysis of a filtering and prioritization process against reference sequences of all protein-coding regions. Many disease-causing mutations are inherited in a Mendelian, common or complex disorders $^{7}$.

In an attempt to identify potential causative variants of early-onset familial AD, three pedigrees with AD family history and severe clinical phenotypes were recruited. Functional and large conserved mutations were extracted by WES. A significant number of risk variants were found, and disease-related single-nucleotide polymorphisms (SNPs) were inferred through a literature search.

There is a high probability of variation at evolutionarily conserved amino acid sites having a substantial effect on protein function ${ }^{8}$. Risk variants extracted via WES were validated in a population-based case-control study using Sanger sequencing, which selectively analyzes sequence regions of interest.

This study suggests a $C D K A L 1$ polymorphism as a novel candidate for the detection of early-onset AD.

\section{MATERIALS AND METHODS}

\section{Patients}

This study was approved by the Chung-Ang University Hospital Institutional Review Board (IRB no. C2015258 (1716), and involved a family group consisting of two affected and two unaffected individuals respectively (Supplementary Fig. 1). Informed consent was submitted by all subjects when they were enrolled. Each family member developed AD before age two and was selected based on SCOring Atopic Dermatitis (SCORAD) score index $(>50)$ and total serum IgE level ( $>1,000 \mathrm{kU} / \mathrm{L})$.

Table 1. Clinical characteristics of study subjects

\begin{tabular}{lcc}
\hline \multicolumn{1}{c}{ Characteristic } & Healthy control & Early-onset AD \\
\hline Total & 61 & 112 \\
Age $(\mathrm{yr})$ & $0.9 \pm 0.7$ & $1.8 \pm 0.8$ \\
Male $(\%)$ & 54.1 & 63.4 \\
Total IgE $(\mathrm{kU} / \mathrm{L})$ & - & $159.5 \pm 294.6$ \\
Eosinophils $\left(\mathrm{mm}^{3}\right)$ & - & $382.0 \pm 422.9$ \\
\hline
\end{tabular}

Values are presented as number only or mean \pm standard deviation. AD: atopic dermatitis, IgE: immunoglobulin E.
One hundred twelve AD patients and 61 healthy controls under age 2 years 9 months were enrolled, and the characteristics of the study cohort are presented in Table 1. To validate the risk of $A D$, Sanger sequencing was performed for ERBB2 and CDKAL 1 polymorphisms.

Peripheral blood samples were obtained from all subjects. We tried to eliminate environmental factors as much as possible by recruiting early-onset cases and confirming risk variants that might affect heredity. All subjects were diagnosed with AD by a dermatologist.

\section{Whole-exome sequencing}

Blood samples were collected in ethylenediaminetetraacetic acid tubes from all subjects, and genomic DNA was isolated using a QIAamp DNA Mini Kit (Qiagen Inc., Valencia, CA, USA). The DNA purity and concentration were measured using a Nanodrop spectrometer (Nanodrop Technologies, Wilmington, DE, USA) and a Qubit fluorometer (Life Technologies, Grand Island, NY, USA). WES was performed using SureSelect Human All Exon V4+UTR $71 \mathrm{Mb}$ (Agilent, Santa Clara, CA, USA), according to the manufacturer's standard protocol. DNA samples were sheared using Covaris (Covaris, Woburn, MA, USA). A paired-end (PE) DNA sequencing library was prepared through shearing, end-repair, A-tailing, peak detection, PE adaptor ligation, and amplification. After the library was hybridized with bait sequences for 24 hours, the captured library was purified and amplified with an index barcode tag. Then, library quality and quantity were measured. Sequencing of the exome library was carried out using the 100-bp PE mode of the HiSeq SBS kit (Illumina, San Diego, CA, USA).

\section{Whole-exome sequencing processing and alignment}

Millions of sequence reads in FASTQ format were mapped to UCSC hg19 of the human assembly using the Burrows-Wheeler Aligner (BWA,v0.7.7) ${ }^{9}$ with "mem" and seed value parameters "- $\mathrm{k} 45$ " to create sequence alignment map (SAM) files with correct mate-pair information for the genome. Picard (v1.92) was then used to convert the SAM files to compressed binary alignment map (BAM) files and then to sort the BAM files by chromosome coordinates. The Genome Analysis Toolkit (v2.3.9Lite) ${ }^{10}$ was used for local realignment process of the BAM files at intervals corresponding to potential insertion and deletion alignment errors. SNPs and indels were annotated using snpEff (v3.6c $)^{11}$, which classified the variants.

\section{Annotation}

The dbNSFP is an integrated database of functional predictions from multiple algorithms (scale invariant feature 
transform [SIFT], Polyphen2, phylogenetic $p$-values [phyloP], PhastCons, 1000 Genome).

Filter 1: SnpEff (http://snpeff.sourceforge.net/index.html) annotates type of variants and predicts their effects on genomic regions (e.g., synonymous, nonsynonymous, missense, nonsense, stop-gain, and frameshift point mutations and indels).

Filter 2: SnpEff represents putative variant impact (High: Splice_Site, Start_Lost, Frame_Shift, and Stop_Gained; Moderate: Non_Synonymous_coding, Codon_Insertion and Deletion; Low: Start_gained, etc.).

Filter 3: SIFT and Polyphen2

SIFT score predicts whether the substitution of amino acids affects protein function.

SIFT prediction is on the basis of the degree of amino acid conservation derived from related sequences through PSI-BLAST. It ranges from 0 to 1 ; a value lower than 0.05 or not significant is considered detrimental, otherwise it is considered tolerable.

The Polyphen2 HDIV score based on HumDiv, i.e., hdiv_prob ranged from 0 to 1 . The scores of prediction were "probably damaging, $0.957 \sim 1$ "; "possibly damaging, $0.453 \sim 0.956$ "; and "benign, $0 \sim 0.452 . "$

Filter 4: The phyloP of dbNSFP reflects the score of evolutionary conservation sites under negative or positive selection over the branches of the phylogenetic tree. A higher score indicates a more conserved site (http://varianttools. sourceforge.net/Annotation/DbNSFP).

Filter 5: PhastCons scores contain multiple alignments of 99 vertebrate genomes to the human genome and predict conserved elements. A high score $(>0.2)$ represents a functionally important genomic region.

Filter 6: The 1000 Genome Project selects variants with minor allele frequencies (MAFs, less than 0.01 or unknown) in the global population.

Filter 7: The in-house Korean variation database at the Theragen Etex Bio Institute selects variants (MAFs, less than 0.02 or unknown).

\section{Sanger sequencing}

Polymerase chain reaction (PCR) amplification of two SNPs was performed at $95^{\circ} \mathrm{C}$ for 10 minutes, followed by 35 cycles at $95^{\circ} \mathrm{C}$ for 30 seconds, $55^{\circ} \mathrm{C} \sim 58^{\circ} \mathrm{C}$ for $30 \mathrm{sec}-$ onds and $72^{\circ} \mathrm{C}$ for 40 seconds, with a final extension at $72^{\circ} \mathrm{C}$ for 90 seconds. The PCR reaction mixtures (total volume $50 \mu \mathrm{l}$ ) contained $25 \mu \mathrm{I}$ of $2 \mathrm{X}$ EF-Taq premix (SolGent, Seoul, Korea), $18 \mu \mathrm{l}$ of distilled water, $2.5 \mu \mathrm{l}$ of oligonucleotide primer $(10 \mathrm{pmol} / \mu \mathrm{l})$, and $2 \mu \mathrm{l}$ of template containing $20 \mathrm{ng}$ genomic DNA. The PCR products were purified using a PCR purification kit (Favorgen, Pingtung, Taiwan) and were sequenced on an Applied Biosystems 3500 DNA sequencer (Applied Biosystems, Foster City, CA, USA) according to the manufacturer's instructions.

\section{Statistical analysis}

Using logistic regression analysis, crude odds ratios (ORs), 95\% confidence intervals (Cls), and associations between $A D$ and the SNPs under investigation were calculated. Associations between polymorphisms (rs77152992 and rs1058808) and AD were assessed using Fisher's exact test.

\section{RESULTS}

\section{Whole-exome sequencing in three families with atopic} dermatitis

We used WES to investigate family-specific candidate variants of three Korean families comprising two AD-affected and two unaffected individuals (Supplementary Fig. 1). To limit our study to genetic influences and minimize environmental factors, we recruited early-onset cases with severe clinical $A D$ phenotypes.

WES was used to sequence the exomes of each individual

Table 2. Effects of CDKAL1 and ERBB2 SNPs and protein function prediction scores

\begin{tabular}{|c|c|c|c|c|c|c|c|c|c|c|c|c|}
\hline Gene & RS\# & Chr & POS & AAC & Type & SIFT* & 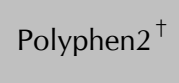 & PhyloP $^{\ddagger}$ & PhastCons ${ }^{\S}$ & Global $^{\|}$ & $\begin{array}{c}\text { East } \\
\text { Asian }\end{array}$ & Korean \\
\hline$C D K A L 1$ & rs77152992 & $6 \mathrm{p} 22.3$ & 21065449 & P409L & cSNP & 0.01 & 0.204 & 5.609 & 1 & 0.06 & 0.11 & 0.149 \\
\hline ERBB2 & rs1058808 & $17 q 12$ & 37884037 & P1170A & cSNP & 0.03 & 0.953 & 3.926 & 1 & 0.49 & 0.48 & 0.425 \\
\hline
\end{tabular}

SNPs: single-nucleotide polymorphisms, Chr: chromosome, POS: position, AAC: single-letter codes for amino acids, cSNP: SNPs in coding regions. *Prediction scores for amino acid substitutions that affect protein function (damaging $<0.05$, tolerance $>0.05$; scores range from 0 to 1$)$. ${ }^{\dagger}$ Prediction of the possible impact of amino acid substitutions $(0.957<$ probably damaging $<1,0.453<$ possibly damaging $<0.956,0<$ benign $<0.452$; scores range from 0 to 1 ). ${ }^{\ddagger}$ Prediction of conserved sites across species; a higher score indicates

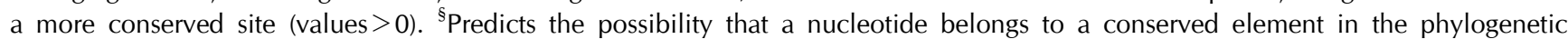
tree (value $>0.2)$. "Global frequency, variants with minor allele frequencies (MAFs) as low as $1 \%$ or with an unknown frequency (value $<0.01$ ). "Korean frequency, variants with MAFs as low as $2 \%$ or in unknown genes (value $>0.02$ ). 
in three families with a history of $A D$, and then this variant data was filtered in different steps (Supplementary Fig. 2). To identify susceptible variants for AD, fast and convenient filters for dominant, recessive and heterozygous models were used. A number of variants were excluded in each filtering step, and conserved and functional variants remained in filters $5 \sim 7$. All variants were counted after each filtering step (Supplementary Table 1 3). Considerable numbers of non-synonymous SNPs that affect a codon via amino acid substitution were observed (data not shown). We focused on functional variants which was common variants (MAF greater than 1\%) in filter 5, rare variants (MAF lower than 1\%) for global population in filter 6 and rare variants (MAF lower than 2\%) for Koreans in filter 7.

Papers related to allergic immune diseases were searched using SNPedia (http://www.snpedia.com/index.php/SNPedia) for putative variants of Filter 5 7 (Supplementary Table $1 \sim 3$ ). We found CDKAL1 (rs77152992) and ERBB2 (rs1058808) non-synonymous SNPs associated with allergic immune diseases like asthma, psoriasis or AD. Although filaggrin SNPs were also detected in WES, they were filtered at a lower step (data not shown).

The rs77152992 variant of CDKAL1 was detected in family $B$ through dominant model analysis. All affected individuals were heterozygous for the identified variants, while unaffected individuals were homozygous for the wild-type allele. The rs1058808 variant of ERBB2 was identified in family $\mathrm{C}$ through compound-heterozygous model analysis. Both the child and the affected father were heterozygous, while the unaffected mother was homozygous for the alt allele (Supplementary Table 4).

CDKAL 1 and ERBB2 SNPs were identified as missense mutations: c.1226C $>$ T, p.Pro409Leu (rs77152992), c.3463 C $>$ G, and p. Pro1170Ala (rs1058808) respectively. rs77152992 was located in chromosome 6p22.3 and rs1058808 was identified in $17 q 12$. The low SIFT scores of the $C D K A L 1$ (0.01) and ERBB2 (0.03) variants predicted high protein damage via amino acid substitution. The high polyphen2 scores of the CDKAL1 (0.204) and ERBB2 (0.953) variants predicted harmful effects on protein structure due to substitution. The phyloP positive scores of the CDKAL1 (5.609) and ERBB2 (3.926) variants indicated that two sites were evolutionarily conserved in multiple alignments of 99 vertebrate genomes to the human genome. The phastcons scores (1) of both sites showed strong conservation in evolutionary events.

The $6 \% \sim 14 \%$ MAF of the CDKAL1 variant and $43 \%$ $49 \%$ MAF of the ERBB2 variant were identified in 1,000 global, 286 East Asians, and 800 Koreans, respectively (Table 2). A case-control study was performed to determine whether rs77152992 and rs1058808 are candidate risk factors for early-onset $\mathrm{AD}$. One hundred twelve patients under age 2 years 9 months with early-onset $A D$ and 61 control subjects were enrolled. A total IgE level of $159.5 \pm 294.6 \mathrm{KU} / \mathrm{L}$ and a total eosinophil level of $382.0 \pm$ $422.9 \mathrm{~mm}^{3}$ for AD patients were used in this study (Table

Table 3. Allele and genotype frequency of CDKAL1 and ERBB2 polymorphisms in 112 Korean AD patients and 61 controls

\begin{tabular}{|c|c|c|c|c|c|}
\hline Gene & SNP & & & Control & $A D$ \\
\hline \multirow[t]{5}{*}{$C D K A L 1$} & rs77152992 & Allele & $\mathrm{C}$ & 102 (83.6) & 207 (9.4) \\
\hline & (c.1226C > T) & & $\mathrm{T}$ & $20(16.4)$ & 17 (7.6) \\
\hline & & Genotype & $\mathrm{CC}$ & $44(72.1)$ & $95(84.8)$ \\
\hline & & & $\mathrm{CT}$ & $14(23.0)$ & $17(15.2)$ \\
\hline & & & TT & $3(4.9)$ & $0(0)$ \\
\hline \multirow[t]{5}{*}{ ERBB2 } & rs1058808 & Allele & $\mathrm{C}$ & $74(60.7)$ & $129(57.6)$ \\
\hline & (c.3463C > G) & & G & 48 (39.3) & $95(42.4)$ \\
\hline & & Genotype & $\mathrm{CC}$ & $21(34.4)$ & 35 (31.3) \\
\hline & & & CG & 32 (52.5) & $59(52.7)$ \\
\hline & & & GG & $8(13.1)$ & $18(16.1)$ \\
\hline
\end{tabular}

Values are presented as number (\%). AD: atopic dermatitis, SNP: single-nucleotide polymorphism.

Table 4. ORs and $95 \% \mathrm{Cls}$ for atopic dermatitis associated with CDKAL1 and ERBB2 polymorphisms in an allele model

\begin{tabular}{llcccc}
\hline \multirow{2}{*}{ Gene } & \multirow{2}{*}{ SNP } & \multicolumn{4}{c}{ Allele } \\
\cline { 3 - 7 } & & Minor allele & Major allele & OR $(95 \% \mathrm{Cl})$ & $p$-value \\
\hline CDKAL1 & rs77152992 & $\mathrm{T}$ & $\mathrm{C}$ & $0.42(0.21 \sim 0.83)$ & 0.0133 \\
ERBB2 & rs1058808 & $\mathrm{G}$ & $\mathrm{C}$ & $1.14(0.72 \sim 1.78)$ & 0.5801 \\
\hline
\end{tabular}

OR: odds ratio, $\mathrm{Cl}$ : confidence interval, SNP: single-nucleotide polymorphism. 
1). Sanger sequencing was performed, and the allele and genotype frequencies of Koreans were determined by direct gene counting. A distinct reduction in rs77152992 minor alleles $(\mathrm{T})$ was observed in $\mathrm{AD}$ patients compared to the control group. The MAF of rs1058808 showed no significant differences between $\mathrm{AD}$ and control groups (Table 3).

ORs with $95 \% \mathrm{Cl}$ were estimated for individual risk alleles. rs77152992 of CDKAL1 was significantly associated with early-onset AD (OR, 0.42; 95\% Cl, $0.21 \sim$ $0.83 ; p=0.0133)$ for (T) allele frequency. rs1058808 of ERBB2 had no correlation with AD (Table 4). The TT + CT genotype of $C D K A L 1$ showed a significantly reduced risk of AD development (OR 0.46; 95\% Cl 0.22 0.99; $p=$ 0.0475) under a dominant model (Table 5). However, there was no significant correlation when using a compound-hetero and recessive model. Conversely, the risk of $\mathrm{AD}$ was 2.16-fold increase $(\mathrm{OR}, 2.16 ; 95 \% \mathrm{Cl}, 1.0 \sim 4.6$; $p=0.0475$ ) when major allele CC genotype was set as a recessive deleterious allele (Table 6). ERBB2 showed no association with AD in any of the three models (Table 5).

The relationship between the genotype of two SNPs and the clinical characteristics (total IgE level and eosinophil count) of AD patients has been confirmed. Total eosinophil count was significantly increased in AD patients with the CC genotype of CDKAL1 ( $p=0.043)$. There was no association between rs77152992 and total IgE levels (Fig. 1). The ERBB2 variant was not related to the clinical characteristics of AD (Supplementary Fig. 3).

\section{DISCUSSION}

AD is a common and complex skin disease associated with both genetic and environmental factors. Early development of AD in infancy carries a higher risk for allergic rhinitis and asthma later in life ${ }^{12}$. In these cases, the detection of risk variants becomes more important for prediction and prevention of AD and allergic march. Lossof-function mutation in the filaggrin gene, a major structural protein in the epidermis, constitutes a well-recognised susceptibility locus for AD. Further, genome-wide association studies (GWAS), including large meta-analyses, have discovered several additional susceptibility loci with genome-wide significance. However, the reported variations only explain a fraction of the overall heritability of $\mathrm{AD}^{13}$.

To identify candidate variants that may affect disease inheritance, a family-based design was used. We chose a family group consisting of four individuals, where one of the parents and the children developed AD at an early age. The family design was also advantageous due to
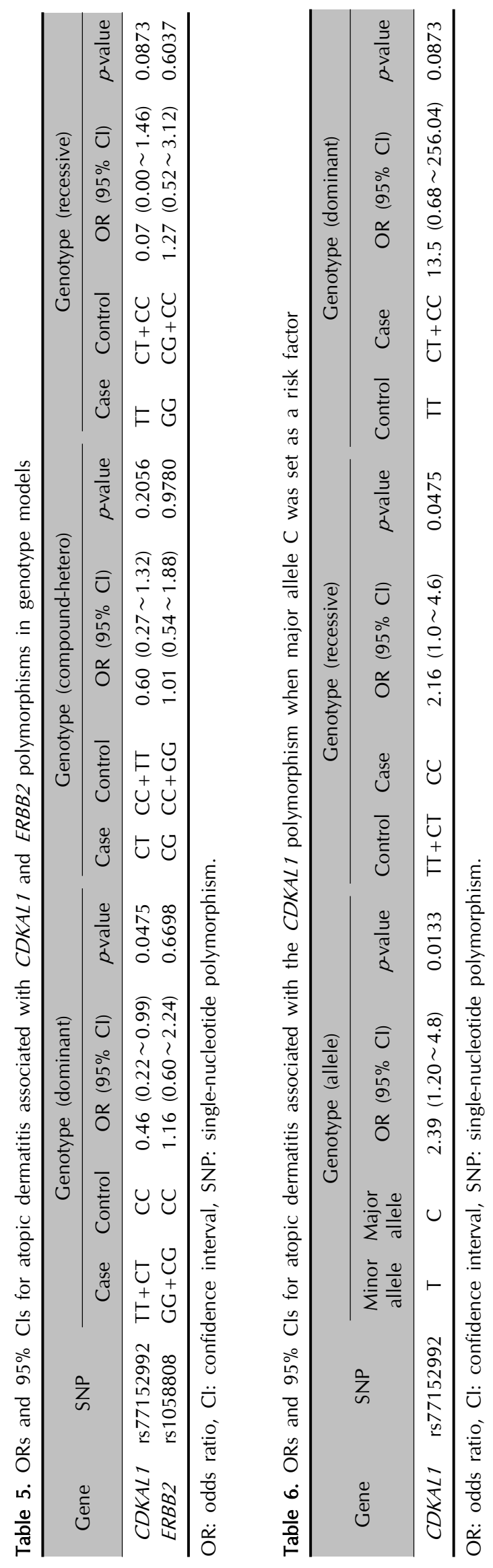

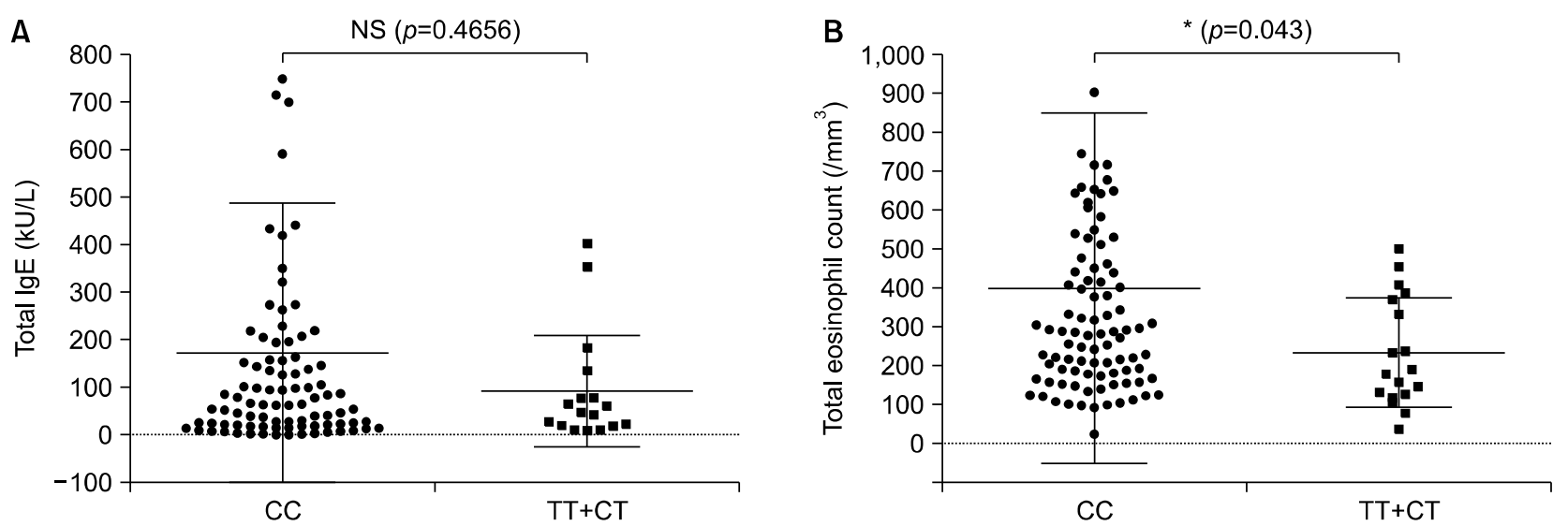

Fig. 1. The relationship of the CDKAL1 polymorphism with total immunoglobulin E (IgE) and eosinophil counts in atopic dermatitis patients. Dot-plot graphs showing concentrations of total IgE levels (A) and eosinophil counts (B) for the CC and TT + CT genotypes. Data are shown as mean \pm standard deviation. NS: not significant. ${ }^{*} p<0.05$ by Mann-Whitney U-test for statistical significance.

cost-effectiveness and identification of hidden variants not detectable in a population-based study like GWAS ${ }^{14}$.

As a method of identifying genetic disease markers, the common disease-common variant (CD-CV) hypothesis was the first dominant theory of common disease in the genetics field. However, the CD-CV hypothesis reached the limit, and the theory that rare variants might have a large effect arose. The issue of which variants have critical effects is still a contentious issue in the field ${ }^{15}$. In this study, WES found family-specific common and rare variants, yet there were no overlapping mutations that occurred in AD patients compared with healthy controls in three families, despite filtering a massive quantity of variants. We confirmed variants related to allergic disease in each family through a literature search and using three models (dominant, hetero and recessive).

The $C D K A L 1$ gene is a member of the methylthiotransferase family located in $6 \mathrm{p} 22.3$. The function of the protein is unknown. The expression of the CDKAL1 gene is absent in primary keratinocytes, whereas abundant expression has been identified in $\mathrm{CD}^{+}{ }^{\mathrm{T}}$ cells ${ }^{15}$. Common SNPs located in the $C D K A L 1$ gene have been linked to increased risk of psoriasis and have been suggested to have an effect on the response to anti-tumor necrosis factor therapies ${ }^{16,17}$. $\mathrm{AD}$ also has immune dysregulation mediated by $\mathrm{T}$ helper cells, although psoriasis is characterized by a $\mathrm{T}$ helper type 1 and/or $\mathrm{T}$ helper type 17 immunological response, whereas acute AD lesions exhibit $T$ helper type 2-dominant inflammation ${ }^{18}$. Functional sequences can be predicted by confirming evolutionary conservation rates across species ${ }^{19}$. Our data suggest that rs77152992 was a strongly conserved protein coding region among 100 vertebrate species. The similar MAF of rs77152992 between 800 Koreans $(14.9 \%)$ and 61 healthy controls (16\%) dem- onstrates the credibility of the data despite the small sample size in this case-control study. A previous study reported that a patient with a chromosome 6 p22.3 deletion had hypereosinophilic syndrome ${ }^{20}$. rs77152992 was located in 6p22.3, and a high eosinophil count has been observed in AD patients with major allele (CC) genotype. It was reported that mean level of peripheral blood eosinophil count was $290.0 \pm 205.7$ total eosinophils $/ \mathrm{mm}^{3}$ in AD patients $(\mathrm{n}=30)$ and $153.3 \pm 113.7$ total eosinophils $/ \mathrm{mm}^{3}$ in healthy control $(n=30)^{21}$. Our data showed $400.9 \pm 449.6$ total eosinophils $/ \mathrm{mm}^{3}$ in $\mathrm{AD}$ patients with $\mathrm{CC}$ genotype and $235.1 \pm 140.6$ total eosinophils $/ \mathrm{mm}^{3}$ in $\mathrm{AD}$ patients with CT + TT genotype. The CC genotype of rs77152992 may be associated with increased eosinophil counts. Thus, The CDKAL1 gene and the CC genotype of rs77152992 may be implicated in early-onset AD.

Mean level of total IgE was $224 \mathrm{kU} / \mathrm{L}(14 \sim 12,013 \mathrm{kU} / \mathrm{L})$ in extrinsic $A D(n=65)$ and $25.2 \mathrm{kU} / \mathrm{L}(0 \sim 4,352 \mathrm{kU} / \mathrm{L})$ in intrinsic $A D(n=38)^{22}$. Our data represented mean level was $172.1 \mathrm{kU} / \mathrm{L}(2 \sim 1,768 \mathrm{kU} / \mathrm{L})$ in AD patients with $\mathrm{CC}$ genotype and $91.77 \mathrm{kU} / \mathrm{L}(10 \sim 401 \mathrm{kU} / \mathrm{L})$ in AD patients with CT + TT genotype. There was no correlation between AD patients with $C C$ and $C T+T T$ genotype group.

The $E R B B 2$ gene is a family member of epidermal growth factor receptor. rs 1058808 was a candidate causal SNP related to asthma in a GWAS, and transmembrane receptor activity was a candidate casual pathway identified through Identify candidate Causal SNPs and Pathways analysis ${ }^{23}$. Although rs1058808 is a functional variant with strong evolutionary conservation and can be damaging to proteins, it was not associated with the risk of early-onset $A D$ pathogenesis in a case-control study. Although the rs1058808 variant was not correlated with AD in our data, Sääf et al. $^{24}$ reported that $E R B B 2$ mRNA was highly ex- 
pressed during keratinocyte differentiation. This protein level showed lower expression in $\mathrm{AD}$ skin compared to healthy controls. Thus, amino acid substitution of rs1058808 may be more likely to impact AD in patients with the mutation.

Taken together, two candidate variants, CDKAL1 (rs77152992) and ERBB2 (rs1058808), were identified in familial genomic data. The high evolutionary conservation of these two SNPs suggested deleterious protein function. Our case-control study suggests that the CC genotype of rs77152992 may be associated with increased eosinophil counts. It may enhance the risk of early-onset AD. Our study strengthens the support for a genetic basis of early-onset Korean AD, and contributes to the development of a diagnostic and predictive tool for this common childhood disease.

\section{ACKNOWLEDGMENT}

This study was supported by a grant from the Korean Healthcare Technology R\&D Project, Ministry of Health \& Welfare, Republic of Korea (HI14C2687). This research was supported by the Chung-Ang University Research Scholarship Grants in 2016.

\section{SUPPLEMENTARY MATERIALS}

Supplementary data can be found via http://anndermatol. org/src/sm/ad-30-276-s001.pdf.

\section{CONFLICTS OF INTEREST}

The authors have nothing to disclose.

\section{REFERENCES}

1. Uehara M, Kimura C. Descendant family history of atopic dermatitis. Acta Derm Venereol 1993;73:62-63.

2. Kryukov GV, Pennacchio LA, Sunyaev SR. Most rare missense alleles are deleterious in humans: implications for complex disease and association studies. Am J Hum Genet 2007;80:727-739.

3. Nutten S. Atopic dermatitis: global epidemiology and risk factors. Ann Nutr Metab 2015;66 Suppl 1:8-16.

4. Li K, Oh WJ, Park KY, Kim KH, Seo SJ. FLG mutations in the East Asian atopic dermatitis patients: genetic and clinical implication. Exp Dermatol 2016;25:816-818.

5. Irvine $A D, M c L e a n W H$, Leung DY. Filaggrin mutations associated with skin and allergic diseases. N Engl J Med 2011;365:1315-1327.

6. Rabbani B, Tekin M, Mahdieh N. The promise of wholeexome sequencing in medical genetics. J Hum Genet 2014;
59:5-15.

7. Pabinger S, Dander A, Fischer $M$, Snajder R, Sperk $M$, Efremova $M$, et al. A survey of tools for variant analysis of next-generation genome sequencing data. Brief Bioinform 2014;15:256-278.

8. Thomas PD, Kejariwal A. Coding single-nucleotide polymorphisms associated with complex vs. Mendelian disease: evolutionary evidence for differences in molecular effects. Proc Natl Acad Sci U S A 2004;101:15398-15403.

9. Li H, Durbin R. Fast and accurate short read alignment with Burrows-Wheeler transform. Bioinformatics 2009;25:17541760.

10. DePristo MA, Banks E, Poplin R, Garimella KV, Maguire JR, Hartl C, et al. A framework for variation discovery and genotyping using next-generation DNA sequencing data. Nat Genet 2011;43:491-498.

11. Cibulskis K, Lawrence MS, Carter SL, Sivachenko A, Jaffe D, Sougnez $C$, et al. Sensitive detection of somatic point mutations in impure and heterogeneous cancer samples. Nat Biotechnol 2013;31:213-219.

12. Garmhausen D, Hagemann T, Bieber T, Dimitriou I, Fimmers R, Diepgen $T$, et al. Characterization of different courses of atopic dermatitis in adolescent and adult patients. Allergy 2013;68:498-506.

13. Bin L, Leung DY. Genetic and epigenetic studies of atopic dermatitis. Allergy Asthma Clin Immunol 2016;12:52.

14. Kazma R, Bailey JN. Population-based and family-based designs to analyze rare variants in complex diseases. Genet Epidemiol 2011;35 Suppl 1:S41-S47.

15. Gibson G. Rare and common variants: twenty arguments. Nat Rev Genet 2012;13:135-145.

16. Quaranta $M$, Burden AD, Griffiths CE, Worthington J, Barker JN, Trembath RC, et al. Differential contribution of CDKAL1 variants to psoriasis, Crohn's disease and type II diabetes. Genes Immun 2009;10:654-658.

17. Coto-Segura P, Batalla A, González-Fernández D, Gómez J, Santos-Juanes J, Queiro R, et al. CDKAL1 gene variants affect the anti-TNF response among Psoriasis patients. Int Immunopharmacol 2015;29:947-949.

18. Miyagaki T, Sugaya M. Recent advances in atopic dermatitis and psoriasis: genetic background, barrier function, and therapeutic targets. J Dermatol Sci 2015;78:89-94.

19. Siepel A, Bejerano G, Pedersen JS, Hinrichs AS, Hou M, Rosenbloom K, et al. Evolutionarily conserved elements in vertebrate, insect, worm, and yeast genomes. Genome Res 2005;15:1034-1050.

20. Ladinsky HT, Elizalde A, Schickler R, Dees PB, Crenshaw ML, Sleasman JW. Hypereosinophilic syndrome and hemimelia in a patient with chromosome 6 p22.3 deletion. Pediatr Allergy Immunol 2014;25:500-503.

21. Jenerowicz D, Czarnecka-Operacz M, Silny W. Peripheral blood eosinophilia in atopic dermatitis. Acta Dermatovenerol Alp Pannonica Adriat 2007;16:47-52.

22. Ott H, Stanzel S, Ocklenburg C, Merk HF, Baron JM, Lehmann S. Total serum IgE as a parameter to differentiate between intrinsic and extrinsic atopic dermatitis in children. Acta Derm Venereol 2009;89:257-261. 
23. Song GG, Lee YH. Pathway analysis of genome-wide association study on asthma. Hum Immunol 2013;74: 256-260.

24. Sääf $A$, Pivarcsi $A$, Winge $M C$, Wahlgren $C F$, Homey $B$,
Nordenskjöld M, et al. Characterization of EGFR and ErbB2 expression in atopic dermatitis patients. Arch Dermatol Res 2012;304:773-780. 
pISSN 1013-9087 • eISSN 2005-3894

Family A
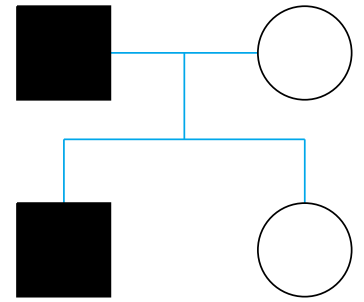

Affected Unaffected
Family B
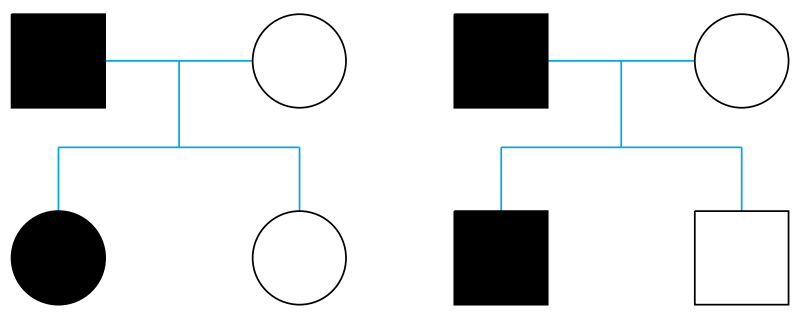

Supplementary Fig. 1. Pedigree diagrams of three families with atopic dermatitis (AD). Squares indicate males, and circles indicate females. Solid symbols represent affected individuals with AD. Unshaded symbols represent unaffected individuals. 


\begin{tabular}{|c|c|}
\hline \multirow{7}{*}{ 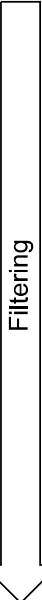 } & Step1: EFFECT \\
\hline & Step2: IMPACT (high and moderate) \\
\hline & 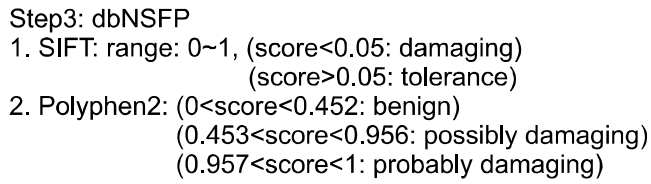 \\
\hline & $\begin{array}{l}\text { Step4: dbNSFP phyloP: (score >0) } \\
\text { *the larger the score, the more conserved the site }\end{array}$ \\
\hline & Step5: PhastCons (score>0.2) \\
\hline & Step6: 1000 Genome (score<0.01)+unknown \\
\hline & Step7: Korean (score $<0.02)+$ unknown \\
\hline
\end{tabular}

Supplementary Fig. 2. Flow chart of the process for filtering variants. SIFT: scale invariant feature transform, phyloP: phylogenetic $p$-values. 

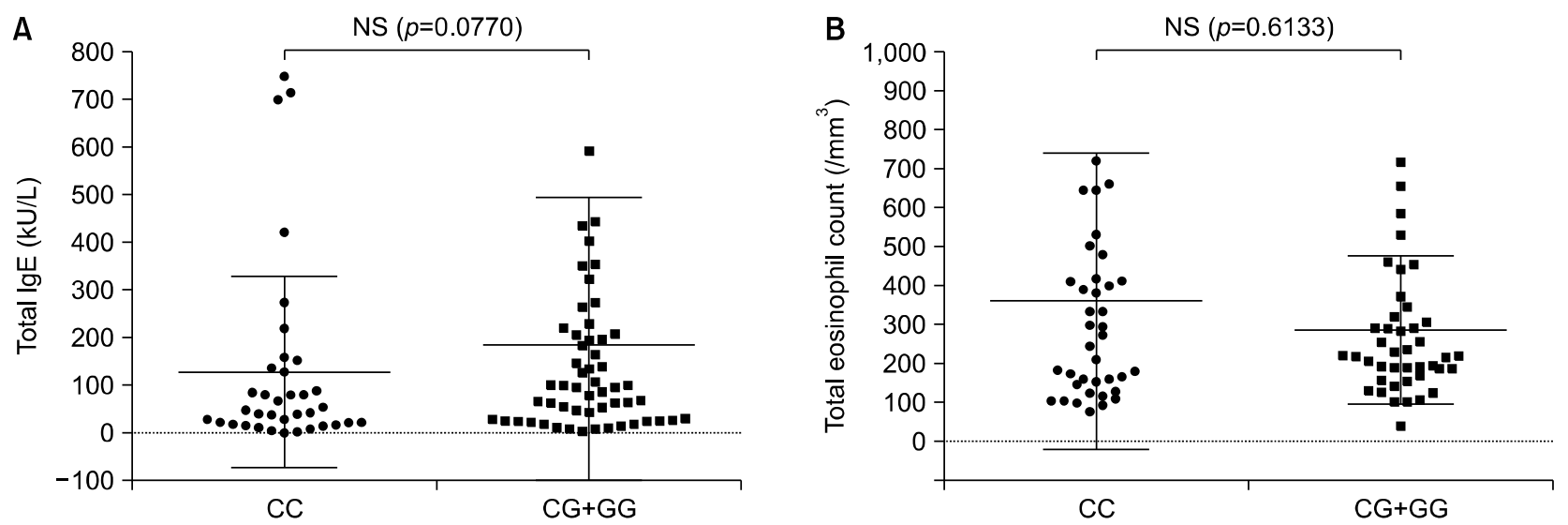

Supplementary Fig. 3. The relationship of ERBB2 polymorphism with total immunoglobulin $\mathrm{E}$ (IgE) and eosinophil count in atopic dermatitis patients. Dot-plot graphs showing concentrations of total IgE level (A) and Eosinophil count (B) with CC and CT+ TT genotype. Data are shown as mean \pm standard deviation. NS: not significant. 
WI Heo, et al

Supplementary Table 1 . The filtered variants in whole-exome sequencing for A family

\begin{tabular}{ccrrrr}
\hline \multicolumn{2}{c}{ Filter system } & & \multicolumn{2}{c}{ Model analysis } \\
\cline { 1 - 2 } \cline { 5 - 5 } Filter step & Filter process & & Dominant & Recessive & Compound hetero \\
\hline Raw & - & 7,523 & 3,420 & 33,866 \\
Filter 1 & EFFECT & 2,799 & 1,306 & 5,227 \\
Filter 2 & IMPACT & 502 & 201 & 949 \\
Filter 3 & SIFT, PolyPhen2 & 278 & 84 & 459 \\
Filter 4 & PhyloP & 234 & 65 & 276 \\
Filter 5 & Phastcon & 200 & 51 & 166 \\
Filter 6 & 1000 Genome & 33 & 3 & 67 \\
Filter 7 & Korean & 31 & 2 & 5 \\
\hline
\end{tabular}

SIFT: scale invariant feature transform, phyloP: phylogenetic $p$-values. 
Supplementary Table 2. The filtered variants in whole-exome sequencing for B families

\begin{tabular}{|c|c|c|c|c|}
\hline \multicolumn{2}{|c|}{ Filter system } & \multicolumn{3}{|c|}{ Model analysis } \\
\hline Filter step & Filter process & Dominant & Recessive & Compound hetero \\
\hline Raw & - & 8,518 & 3,263 & 36,806 \\
\hline Filter 1 & EFFECT & 3,635 & 1,334 & 5,176 \\
\hline Filter 2 & IMPACT & 639 & 201 & 929 \\
\hline Filter 3 & SIFT, PolyPhen2 & 332 & 75 & 406 \\
\hline Filter 4 & PhyloP & 282 & 63 & 262 \\
\hline Filter 5 & Phastcon & 213 & 52 & 176 \\
\hline Filter 6 & 1000 Genome & 77 & 4 & 66 \\
\hline Filter 7 & Korean & 69 & 3 & 7 \\
\hline
\end{tabular}

SIFT: scale invariant feature transform, phyloP: phylogenetic $p$-values. 
WI Heo, et al

Supplementary Table 3. The filtered variants in whole-exome sequencing for $C$ families

\begin{tabular}{|c|c|c|c|c|}
\hline \multicolumn{2}{|c|}{ Filter system } & \multicolumn{3}{|c|}{ Model analysis } \\
\hline Filter step & Filter process & Dominant & Recessive & Compound hetero \\
\hline Raw & - & 8,147 & 3,985 & 36,131 \\
\hline Filter 1 & EFFECT & 3,410 & 1,646 & 4,389 \\
\hline Filter 2 & IMPACT & 586 & 243 & 761 \\
\hline Filter 3 & SIFT, PolyPhen2 & 319 & 88 & 275 \\
\hline Filter 4 & PhyloP & 278 & 75 & 181 \\
\hline Filter 5 & Phastcon & 209 & 52 & 112 \\
\hline Filter 6 & 1000 Genome & 86 & 6 & 53 \\
\hline Filter 7 & Korean & 69 & 2 & 11 \\
\hline
\end{tabular}

SIFT: scale invariant feature transform, phyloP: phylogenetic $p$-values. 
Supplementary Table 4. The genotype of CDKAL1 and ERBB2 in three family

\begin{tabular}{|c|c|c|c|c|c|c|}
\hline \multirow{2}{*}{ Family } & \multirow{2}{*}{ Gene } & \multirow{2}{*}{ Model } & Mother & Father & Child1 & Child2 \\
\hline & & & Unaffected & Affected & Affected & Unaffected \\
\hline A & $\begin{array}{l}\text { CDKAL1 } \\
\text { ERBB2 }\end{array}$ & & & & & \\
\hline B & $\begin{array}{l}\text { CDKAL1 } \\
\text { ERBB2 }\end{array}$ & Dominant & $\mathrm{CC}(0 / 0)$ & $\mathrm{CT}(0 / 1)$ & $\mathrm{CT}(0 / 1)$ & CC $(0 / 0)$ \\
\hline $\mathrm{C}$ & $\begin{array}{l}\text { CDKAL1 } \\
\text { ERBB2 }\end{array}$ & Compound heterozygous & GG $(1 / 1)$ & CG $(0 / 1)$ & CG $(0 / 1)$ & $\mathrm{CG}(0 / 1)$ \\
\hline
\end{tabular}

